\title{
Papel del trabajo de campo en el proceso de planteamiento de problemas de investigación en torno a temas Ambientales. Estudio de caso.
}

\section{The role of the field work in the process of research problems approach about environmental topics.}

Por: Bernal Daissy Lisbeth; Cruz Diana Patricia ${ }^{1}$

Resumen: El papel del trabajo de campo en el proceso de planteamiento de un problema de investigación, que esté relacionado con temas ambientales es de gran importancia, ya que es necesario establecer un contacto directo con el objeto de estudio para poder observar y participar de la dinámica ambiental que este presenta, dinámica que no siempre se hace evidente en documentos bibliográficos. La experiencia del trabajo de campo permite al investigador conocer un contexto donde se pueden aplicar conceptos en la realidad y de esta manera ampliar su visión frente al objeto de estudio, por ejemplo, el hecho de que en dichos contextos habite una comunidad especifica, que es parte crucial para el desarrollo de la investigación.

En este caso el objeto de estudio es el municipio de Manaure - Guajira, que presenta una dinámica ambiental particular, pues el mayor porcentaje de la comunidad residente pertenece a la etnia wayuú; la metodología empleada tuvo un enfoque cualitativo de investigación, y se utilizaron algunas técnicas etnográficas como la observación participante, el diario de campo y la entrevista no dirigida; el trabajo de campo se realizó al final del mes de enero y comienzos de febrero del año 2008, el estar allí permitió reconocer que los habitantes ven la escasez de agua como una problemática que afecta su relaciones con el entorno. Al momento de plantear el problema de investigación se retomó este aspecto y otros, como la pertinencia del territorio para la comunidad, el problema de residuos sólidos, etc., que no fueron evidentes en la documentación previa, y por esto se tienen en cuenta para plantear el problema de investigación. Además, esta experiencia aporta en gran medida a la formación docente permitiéndole enfrentarse a realidades diferentes a la cotidiana.

Palabras clave: trabajo de campo, investigación, ambiente, territorio.

Abstract: The fieldwork role in the process of approaching a research problem that deals with environmental topics is very important, since it is necessary to establish a direct contact with the study object in order to observe and

${ }^{1}$ Estudiantes de licenciatura en biología de la Universidad Pedagógica Nacional, $\mathrm{X}$ semestre. daissylisbeth@yahoo.es, dianapatriciacruz@hotmail.com. 
participate in the environmental dynamics that this presents, that is not always is evident in bibliographic documents.

The fieldwork experience allows the investigator to know a context where concepts can be applied in the reality, and in this way to increase his view on the study object, for example, the fact that in these contexts lives a specific community, is a crucial part for the development of the research.

In this case, the study object is the Manaure municipality - Guajira, which presents a particular environmental dynamics, because the major percentage of the resident community belongs to the Wayuu ethnic group. The implemented methodology had a qualitative research approach and some ethnographic techniques like participant observation, the field diary and non directed interviews were used. The field work was carried out at the end of February in 2008. We could recognize that the inhabitants consider water shortage like a problem that affects their relationships with the environment. At the moment of setting out the research problem this aspect and others such as the territory pertinence for the community, the solid waste, etc, that were no evident in the former documentation, were taken into account. Besides, this experience contributes to teaching education to a large extent, allowing them to face different realities from the daily ones.

Key words: field work, research, environment, territory.

\section{INTRODUCCIÓN.}

El planteamiento de problemas de investigación que integre aspectos ambientales, requiere un contacto directo con el contexto de interés, ya que el estudio de un ambiente específico implica conocer la dinámica que se establece entre los sistemas sociales y biofísicos, lo que permite evidenciar aspectos de gran importancia que no se encuentran en documentaciones previas, es decir, no basta informarse acerca de un lugar y su dinámica respecto al ambiente, es necesario estar allí pues esto posibilita al investigador identificar diferentes posturas críticas y situaciones que no siempre se hacen evidentes en una revisión bibliográfica.

En este caso, se planteó un problema de investigación teniendo en cuenta el contexto de Manaure - Guajira, conocido como un bello lugar donde se extrae la sal, y donde habita parte de la comunidad de indígenas Wayuú, de los que la mayoría de veces, se piensa son grupos aislados y poco modernos. Durante el trabajo de campo estos supuestos cambian, pues la comunidad ha tenido un gran contacto con la sociedad nacional, de tal manera que ha apropiado diferentes formas de pensar y actuar; el estar allí evidencia la existencia de problemas ambientales relacionados con el agua y la basura, que afectan las dinámicas ambientales y además devela posibles causas y su repercusión en una comunidad como esta. El trabajo en campo contribuye a la formación pedagógica y disciplinar necesaria para afrontar las múltiples realidades que son susceptibles de ser investigadas y por lo tanto es fundamental al plantear la elaboración de problemas de dichas investigaciones. Es importante resaltar que el trabajo con una comunidad, en este caso los Wayuú, debe ir más del simple reconocimiento de situaciones, es necesario involucrarla en la investigación, de tal manera que se construya a partir de intereses comunes. 
¿Cuál es la importancia del trabajo de campo en el planteamiento de problemas de investigación en torno a temas ambientales?

Para darle una solución a esta pregunta debemos tener en cuenta, en primera medida, el significado que tiene el trabajo de campo, el cuál implica el reconocimiento de un contexto de interés, donde es importante identificar la comunidad que en él se desenvuelve, y en este caso, para incluir la dimensión ambiental, las relaciones que se establecen con el entorno. Pero también implica involucrarse en este contexto, es decir, tener un contacto directo para poder dilucidar y dimensionar las dinámicas ambientales que se dan en éste, como afirma la Universidad Nacional de Colombia (IDEA, 1996), "el trabajo de campo se constituye en la posibilidad de compartir y participar de la cotidianidad, las formas de relacionarse y la comunicación con las comunidades que constituyen los grupos observados"(Universidad Nacional De Colombia. IDEA, 1996; 69). Se entiende ambiente como un sistema en el que interactúa lo biofísico y lo social (UNESCO, 1977), dichas interacciones están mediadas por la cultura (Bermúdez, 2005), y se caracteriza porque intervienen elementos económicos, tecnológicos, políticos, ecológicos, históricos, etc., (Sauve, 1994) en un espacio y tiempo determinado (Bermúdez, 2005).

Se puede entender la cultura como "un proceso histórico dentro del cual las sociedades se construyen a sí mismas en su interacción con otras; con formas de entender e interpretar la realidad y de organización para vivirla cotidianamente (...) articula una red continua de interacciones, donde se intercambia, se representa, se imagina y se pone en escena toda clase de objetos materiales, de ideas y de relaciones personales y sociales. Es dinámica, cambiante, responde a las condiciones cambiantes del contexto que dan significado de maneras diversas sus coyunturas y sus entornos" (Serje, 2002; 317), evidenciando así, que la historia, el contexto y las interacciones personales y sociales también son elementos relevantes para estudiarla. Es importante mencionar que para Serje "el ambiente siempre está inmerso en la manera como se conciben las sociedades, las culturas y su historia" (Serje, 2002; 317), siendo así la cultura un elemento determinante para entender un sistema ambiental dinámico y cambiante en el tiempo.

Para poder establecer y comprender de una mejor manera estas dinámicas, se hace necesaria una previa documentación acerca de éste contexto, pues ésta permite ampliar el panorama y establecer posibles relaciones. Según Aguirre (1995), antes de iniciar el trabajo de campo es necesaria "una documentación bibliográfica que nos ponga al tanto del tema a abordar y facilite una puesta a punto de principios teóricos que orienten la investigación (...) se puede echar mano de algunas obras que pretendan dar una visión genérica de las sociedades a estudiar" (Aguirre, 1995; 122)

En este caso, el contexto de interés es la Guajira, donde se pretendía identificar una problemática que permitiera el planteamiento de un problema de investigación; decimos identificar, ya que no es posible establecer un problema de investigación sin involucrarse en el contexto, y de ser así, implica muchas modificaciones posteriores. De allí, la importancia que Bourdieu y Wacquant, le atribuyen al papel de la práctica en un trabajo de investigación y a la construcción del objeto de estudio donde "lo que cuenta en realidad, es el rigor en la construcción del objeto" (Bourdieu \& Wacquant, 2005: 308), es decir, para la 
construcción del objeto de estudio se requiere de constancia y rigurosidad, sobre todo cuando se esta trabajando en campo.

De esta forma, se realizó una previa documentación rigurosa del sector incluyendo aspectos biofísicos tales como el clima, la vegetación representativa, las épocas de lluvia, y características generales de la región, además se tuvo en cuenta estudios antropológicos que estaban enfocados en la comunidad residente, constituida por los indígenas wayuú. Algunos autores consultados fueron Correa y Vásquez (2000), Correa (1993) y Vergara (1987). Entre las situaciones descritas que develaban un gran interés y por lo tanto eran susceptibles de ser tenidas en cuenta para ser estudiadas, se resaltaban la explotación del carbón en el Cerrejón, el desarrollo de actividades cotidianas en torno a la dinámica de lluvias, la importancia de el pastoreo, de el intercambio de propiedades, de los saberes tradicionales, la carencia del recurso hídrico, entre otros, que hacen parte de la dinámica ambiental de la zona. Sin embargo, los registros encontrados no son actuales, lo que le confiere mayor importancia al trabajo de campo.

Con base en esto, siendo así la cultura un elemento determinante para entender un sistema ambiental se retoma el concepto de cultura entendida como las concepciones que influyen en representaciones sobre el ambiente y desde aquí se plantea un posible problema de investigación dirigido a la indagación acerca de dichas concepciones en la comunidad wayuú; así mismo, la cultura ambiental también se define como "el reconocimiento del paso del ser humano por la vida y su ambiente, por lo tanto está en constante cambio. Es el producto de la acción individual y colectiva de los seres humanos. La cultura ambiental debe ser reconocida como una construcción constante que refleja el uso de los recursos naturales por el ser humano, y su grado de responsabilidad hacia el entorno" (Mata, 2004), definición que está ligada al concepto de ambiente y cultura, ya que para poder dilucidar la cultura ambiental se debe reconocer la dinámica ambiental, lo que incluye reconocer la cultura como mediadora de las interacciones que se dan en dicha dinámica.

$\mathrm{Al}$ estar la comunidad constituida por indígenas, debe haber un reconocimiento de las características del método etnográfico importantes en el desarrollo del trabajo de campo, y a partir de esta documentación, se evidenció, que en el devenir de éste método, se establece un constante debate en la relación entre sujeto - objeto, donde se resalta una visión contemporánea en el que se rompe con dicha relación de dominación - explotación y se propone un constante diálogo de saberes, reconociendo las formas de conceptualizar de la comunidad para construir conocimiento desde y con ellos. Como afirma Vasco: "el verdadero objeto de conocimiento es la unidad expresada en la relación entre esas sociedades diferentes, pero ya desde hace mucho tiempo imposibles de desligar, de considerar como independientes las unas de las otras. Y mientras no se asuma tal realidad y con base en ella se replanteen los procesos de conocimiento, la cientificidad de los vigentes nos está negada. No se trata pues de integrarnos voluntariamente al objeto, se trata de aceptar que objetivamente somos parte de él"' (Vasco, 2002).

Además, se tuvo en cuenta el concepto de reflexividad que propone Guber (2001), retomando a Bourdieu, inherente al trabajo de campo como "el proceso de interacción, diferenciación y reciprocidad entre la reflexividad del sujeto cognoscente - sentido común, teoría, modelos explicativos- y la de los actores o sujetos/objetos de investigación (Guber, 2001:53), 
contribuyendo a la comprensión de la relación entre el investigador y la comunidad. Se empleó el enfoque cualitativo de investigación el cual se caracteriza por "buscar dimensiones no conocidas o poco conocidas de un hecho social. Estas dimensiones se buscan también a partir de la forma cómo viven y entienden ese hecho los grupos afectados por él. Entre los planteamientos se considera el contexto y su historia, las relaciones e intercambios sociales, las representaciones sociales y el lenguaje" (Badilla, 2006; 44), y se recurrió a algunas técnicas etnográficas para la recolección de la información como la observación participante, el diario de campo y la entrevista no dirigida.

Con todas estas ideas se llegó al lugar de trabajo. Durante el trabajo de campo, estos supuestos cambian, cuando el investigador participa en un contexto como éste, pues sus habitantes han tenido un gran contacto con la sociedad nacional de tal manera que han apropiado diferentes maneras de pensar y actuar; el estar allí, permite evidenciar que los posibles problemas de interés encontrados en la documentación, no tenían la misma relevancia en la realidad, o van más allá de lo que un texto puede hacer evidente, como lo afirma la Universidad Nacional de Colombia (IDEA, 1996), el trabajo de campo es el espacio real en que nos encontramos verificando nuestras representaciones y los aprendizajes que unos y otros creen haber comprendido con el trabajo previo (Universidad Nacional De Colombia. IDEA, 1996). Es así que el investigador debe enfrentarse a situaciones no conocidas, ni esperadas, y hacer uso de los referentes que tiene del contexto para relacionarlo con la realidad, coincidiendo con los planteamientos de Guber (2001) “¿Para qué el campo? Porque es aquí donde modelos teóricos, políticos, culturales y sociales se confrontan inmediatamente - se advierta o no - con los de los actores" (Guber, 2001: 53).

De esta manera, se puede evidenciar la dinámica ambiental allí presente, donde cobra gran importancia la interacción del sistema biofísico con el social determinado por lo cultural, sus características (aspectos económicos, políticos, filosóficos, etc.) y su transformación en el tiempo. Desde este punto de vista, la interacción constante con la comunidad es una forma de reconocer dicha dinámica, que servirá para plantear problemas de investigación acordes al contexto.

Es así que, el Municipio de Manaure, presentó varias situaciones que cobraban relevancia para la comunidad y para los investigadores, de las cuales se resaltan la importancia del recurso hídrico para la comunidad, ya que ésta es muy escasa, ésta situación se evidencia en la afirmación que hace Darwin un indígena Wayuú que habita en la región "como va hacer eso que en tu casa hay un solo baño, hay un solo balde y no haya agua, con que van hacer la comida, que voy a tomar yo, donde el agua es fuente de vida, el agua que para el ser humano es todo y aquí en Manaure nos privan de ese derecho, del derecho del agua, no tenemos agua aquí, no tenemos ninguna clase de acceso al agua". Además esto ha permitido el ingreso de organizaciones que ven la situación como una oportunidad para lucrarse; así lo afirma Valencia Ipuana, un habitante Wayuú "por eso aquí hay un atraso bastante especial en todos los aspectos económicos, en la calidad de vida, todo, entonces vienen las instituciones hacen sus vainas y no se ven".

Así mismo, la presencia de residuos sólidos constituye otra problemática, que desde el punto de vista del investigador, requiere un tratamiento específico para evitar la 
proliferación de las enfermedades y contaminación de suelo y agua, sin embargo el desconocimiento del tema, ha generado su acumulación; los habitantes del sector urbano, afirman que, "aquí (refiriéndose a Manaure) no hay barrido de calles, no hay lavamiento de las vías públicas están las canecas pero están como de lujo porque nadie las utiliza, hay problemática grave con los desechos sólidos y es que están tirados en la calle y son arrastrados a los estanques y hay unos animales que se alimentan y toman de esa agua (Eliécer Epinayu) "...es que Manaure tiene muchas basuras porque no todas llegan al botadero (Yesuri Arpushaina)" "... es que aquí en Manaure, se encuentran muchos lugares en donde se acumula la basura, además hay que tener en cuenta los vientos que se la pasan revolviendo todo, y es que nosotros nunca nos acordamos de los paisanos que viven lejos porque ellos sí que están mal, llega ahí la basura, y las aguas sucias, y así se las tienen que tomar " (Guillermo Aguilar), es de está forma que la comunidad reconoce la existencia de esta problemática, sin embargo no se hace evidente en las actividades cotidianas una responsabilidad y conciencia frente a esta.

Siendo este el panorama de Manaure, se evidencia que la dinámica ambiental presenta problemas que afectan sus interacciones, es por ello que se decide trabajar en torno a las problemáticas ambientales mencionadas anteriormente, relacionando los intereses que posee la comunidad y los de los investigadores; gracias a la experiencia del trabajo de campo, se logro evidenciar la importancia del territorio para la comunidad wayuu, por ejemplo, en palabras de Genaro Epinayú, ingeniero ambiental, miembro de la comunidad de Jirtud "...Muchas veces hay lideres que manejan la comunidad, y entonces ellos convencen al resto, pero desconociendo de pronto los decretos las leyes que nos amparan a nosotros los territorios indígenas, entonces la persona que decidió por nosotros de pronto pensó que nosotros desconocíamos (refiriéndose a la construcción de una represa que compromete gran parte de la ranchería), o el nos ignoró básicamente, eso es lo que sucede, entonces hay instituciones que tomas atribuciones por los demás..."- en ese momento varios miembros de distintas comunidades aledañas al arroyo Limón, se encontraban haciendo campamento temporal en el sitio donde se construiría la represa, como forma de protesta. Es por ello que se tuvo en cuenta este concepto para el replanteamiento del problema de investigación.

En este sentido se entiende territorio como "formas de ocupación y poblamiento, modos de apropiación a través de formas de trabajo, autoridad y pensamiento, divisiones internas, fronteras y sitios históricos y de otra indole, actividades que sobre él se desarrollan, modalidades de recorrerlo, creencias y concepciones asociadas, todo ello constituye ese vasto conglomerado de relaciones sociales que hacen de un espacio sobre la tierra el territorio de una sociedad en un momento de su historia, siendo uno de los elementos básicos de su identidad frente a las demás, Concebimos el territorio como el conjunto de relaciones que una sociedad establece con su espacio geográfico en un momento dado de su existencia (Vasco 2002; 202).

Así, para las comunidades indígenas, este concepto cobra importancia ya que las relaciones que establecen con él han sido rotas, lo que los ha llevado a una lucha constante por recuperarlo y defenderlo. Sin embargo, el territorio se conserva en diferentes elementos de la vida social, por ejemplo el lenguaje, o básicamente lo inmaterial, "es mantener la vigencia de la territorialidad dentro de la vida comunitaria y constituye, como se ha podido constatar, algo importante y que fortalece y fundamenta la lucha de esas sociedades por la recuperación del espacio físico perdido" (Vasco 2002;126). 
A partir de toda esta experiencia, se replantea el problema de investigación que se traía antes de realizar el trabajo de campo. Actualmente, el problema planteado apunta a reconocer la incidencia de la problemática ambiental asociada al manejo del agua y residuos sólidos en los procesos de constitución territorial en el municipio de Manaure (Departamento de la Guajira), intentando recoger los intereses tanto de la comunidad como de los investigadores, siendo consecuentes con los conceptos retomados.

Cabe resaltar que el trabajo en campo contribuye a la formación pedagógica y disciplinar necesaria para afrontar las múltiples realidades que son susceptibles de ser investigadas y por lo tanto, es fundamental al plantear la elaboración de problemas de dichas investigaciones. Además, el papel de la práctica es fundamental para la enseñanza de cualquier tipo de conocimiento, "historiadores y filósofos de la ciencia, y especialmente los científicos mismos, han observado a menudo que buena parte del oficio del científico, se adquiere por medio de modos de transmisión que son enteramente prácticos (Bourdieu \& Wacquant, 2005; 311), pero sin caer sólo en el activismo sin un objetivo a perseguir, no se puede dejar de lado la teoría.

\section{CONCLUSIONES}

El planteamiento de problemas de investigación que integren problemas ambientales, requiere un contacto directo con el contexto de interés, ya que el estudio de un ambiente específico, implica conocer la dinámica que se establece entre los sistemas sociales y biofísicos, lo que permite evidenciar aspectos de gran importancia que no se encuentran en documentaciones previas, es decir, no basta informarse acerca de un lugar y su dinámica respecto al ambiente, es necesario estar allí pues eso posibilita al investigador identificar diferentes posturas críticas y situaciones que no siempre se hacen evidentes en una revisión bibliográfica.

El trabajo de campo permite ajustar y construir conceptos pertinentes para el desarrollo de un problema de investigación.

Es pertinente resaltar, que el trabajo con una comunidad, en este caso los wayuú, debe ir más allá del simple reconocimiento de situaciones, es necesario involucrarla en la investigación, de tal manera que se construya a partir de intereses comunes. Hay que mencionar que para trabajar con esta comunidad se debe tener un acercamiento más profundo con algunos de sus habitantes de modo que sean quienes permitan el contacto con otros habitantes y así evitar que la lengua, en este caso el wayuunaiki, sea un obstáculo, y que se presenten conflictos por ser desconocidos los que llegan a sus residencias y sus intenciones.

En el momento de plantear un problema de investigación que requiera trabajo de campo, es fundamental tener en cuenta la posibilidad de volver al lugar de estudio, ya que esto puede volver a generar nuevos elementos y conceptos que inicialmente parecían poco relevantes.

El trabajo de campo contribuyó a nuestra formación docente en tanto que permitió adquirir experiencia en el trabajo con comunidades, enfrentarse a realidades distintas a las 
cotidianas, es decir, que presentan una dinámica ambiental diferente donde lo cultural determina sus interacciones. Además se hizo evidente el sistema ambiente en una situación particular.

Además el conocimiento de la dinámica ambiental particular de la zona permite evidenciar elementos que son reconocidos para llegar a plantear una educación ambiental contextual y no sólo en el ámbito escolar, sino involucrando a la comunidad y esto constituye un gran aporte a la formación docente, ya que la educación trasciende el aula.

El trabajo de campo es fundamental en la formación y la práctica docente ya que este permite establecer un acercamiento directo además del reconocimiento experiencial de una comunidad, estableciendo actividades y tareas fundamentales para el desarrollo de un trabajo colectivo como lo es el planteamiento y desarrollo de una investigación.

Es necesario incluir en los programas de formación docente el trabajo de campo como una herramienta útil para llegar a plantear problemas de investigación que contribuyan al enriquecimiento de los futuros maestros, y poder llegar a establecer esta herramienta como parte del que hacer docente, es decir, incluirla en su práctica en los lugares en los que se desenvuelve cotidianamente.

El contexto escogido como objeto de estudio, La Guajira, presenta una gran riqueza tanto biofísica como social, en donde es posible el planteamiento de múltiples problemas que son susceptibles de ser investigados.

\section{Bibliografía}

- AGUIRRE, A. 1995. Etnografía. Metodología cualitativa en la investigación sociocultural. Alfaomega Grupo Editor S.A. México. 376 p.

- BADILLA, L. 2006. Fundamentos del paradigma cualitativo en la investigación educativa. Universidad de Costa Rica. En: Revista de Ciencias del Ejercicio y la Salud. Vol. 4, № 1, p. 42 - 51.

- BERMÚDEZ, O. 2005. Representaciones sociales y mapas mentales del campus universitario. Primera etapa. Facultad de artes. Editorial Antropos. Bogotá. 135 p.

- UNIVERSIDAD NACIONAL DE COLOMBIA. Instituto de Estudios Ambientales (IDEA). 1996. Visiones del medio ambiente a través de tres etnias colombianas. Equipo de investigación Olga María Bermúdez Guerrero. Universidad Nacional de Colombia. Instituto de Estudios Ambientales. Bogotá. 98 p.

- BOURDIEU, P.; \& WACQUANT, L. 2005. Una invitación a la sociología reflexiva. Siglo XXI Editores Argentina S.A. Argentina. 430 p.

- CORREA, H. Y VÁSQUEZ, S. 2000. Los wayuú, entre Juyá ("El que llueve"), Mma ("la tierra") y el desarrollo urbano regional. En: Instituto Colombiano de Cultura Hispánica. Geografía Humana de Colombia. Nordeste Indígena (Tomo II).Bogota. Publicación digital en la página Web de la biblioteca Luis Ángel Arango del Banco de la República. $<$ http://www.lablaa.org/blaavirtual/geografia/geograf2/wayuu12.htm> [Búsqueda realizada el 16 de febrero de 2008].

- CORREA, H. 1993. Los wayuú: pastoreando el siglo XXI. En: Encrucijadas de Colombia Amerindia. ICANH. Instituto Colombiano de Antropología e historia. Bogotá. 334 p. 
- GUBER, R. 2001. La etnografía: Método, campo y reflexividad. Grupo Editorial Norma. Bogotá. Colombia. 146 p.

- MATA, A. 2004. Transformación de la cultura ambiental desde la docencia universitaria. En: Revista Biocenosis. Vol.18 (1-2) 2004. Publicación digital en la página web $<$ http://www.uned.ac.cr/biocenosis/articulosVol18/Vol1817.pdf $>$ [Búsqueda realizada el 2 de Junio de 2009].

- SAUVÉ, L. 1994. Exploración de la diversidad de conceptos y de prácticas en la educación relativa al ambiente. En: Seminario Internacional: La dimensión ambiental y la escuela memorias -Serie documentos especiales Ministerio de Educación Nacional, Bogotá.

- SERJE, M., SUAZA, M. VARGAS, C., PINEDA R. 2002. Palabras para desarmar. Una aproximación crítica al vocabulario del reconocimiento cultural en Colombia. Bogotá: Instituto Colombiano de Antropología e Historia, ICANH. Ministerio de Cultura.

- UNESCO. 1977. Conferencia Intergubernamental Sobre Educación Ambiental. Tbilisi, Geórgia.

- VASCO, L. 2002. Entre selva y páramo: viviendo y pensando la lucha india. ICAN. Bogota. 503 p.

- VERGARA, O. 1987. Guajiros. En: Introducción a la Colombia Amerindia. Instituto Colombiano de Antropología. Edición original: Bogota: Editorial Presencia. Publicación digital en la página web de la Biblioteca Luis Ángel Arango del Banco de la República. $<$ http://www.lablaa.org/blaavirtual/antropologia/amerindi/guajiros.htm> [Búsqueda realizada el 16 de febrero de 2008]. 